\title{
Criterios \\ La encuadernación de los libros de coro: las cubiertas de los cantorales de la Abadía del Sacromonte de Granada
}

Javier Bueno Vargas

Dpto. de Pintura de la Universidad de Granada

\section{Resumen}

Los libros de coro constituyen una tipologia muy especifica dentro de la historia del libro. Su uso en diferentes instituciones religiosas condicionó tanto su formato, estructura y encuadernación, como los materiales con que se realizaron y los acabados que éstos recibieron. Hoy en dia destacan por el mal estado de conservación que en general presentan al haber quedado almacenados en vitrinas 0 archivos 0 en sótanos, buhardillas o armarios. En este artículo nos centramos en las particularidades de una colección de cantorales cuyo núcleo principal se confeccionó durante los siglos XVII y $\mathrm{XVIII}$, analizando tanto los materiales como los acabados y sistemas de protección de la encuadernación. Son éstos elementos fundamentales en esta tipologia y su estudio suele quedar relegado cuando se abarca el análisis de alguna de estas colecciones. Sirva este trabajo para dedicarles la atención que merecen, no sólo como obras de arte que son, sino por su valor antropológico como objetos fundamentales para entender a la sociedad que los creó y los consideró elementos indispensables en los ritos religiosos.

\section{Palabras clave}

\section{Patrimonio documental}

Libro de coro

Cantoral

Técnicas artisticas

Encuadernación

Elementos decorativos

Bollón

Esquinera

Abadia del Sacromonte

Granada

Siglos XVII-XX

\section{Introducción}

La Abadía del Sacromonte es una institución religiosa que surgió en Granada a comienzos del siglo XVII, a partir del hallazgo de los supuestos restos óseos de San Cecilio y de sus primeros discipulos. Hoy dia, de entre los numerosos bienes que custodia, destaca una importante colección de casi un centenar de libros de coro, que fueron indispensables para cumplir una de las obligaciones diarias para las que se creó esta Institución, el Culto Divino; los cantorales fueron realizados en diferentes talleres fundamentalmente de Andalucía entre los siglos XVII-XVIII, aunque los hay también de hasta principios del siglo XX.

Confeccionados con diversos materiales, en un primer acercamiento destacan por su gran tamaño y sus particulares revestimientos (en pieles coloreadas a veces decoradas) y por los elementos presentes en la cubierta (esquineras, bollones y cierres), características exclusivas de estos volúmenes; si los abrimos nos encontraremos hojas de pergamino (en los más antiguos, aunque también las hay de papel en los realizados en el siglo XX), con ilustraciones multicolor (iniciales, orlas y peones). Del conjunto se han analizado exhaustivamente $45^{1}$ en una primera fase del estudio de la colección, lo que nos ha permitido determinar ciertas particularidades de este tipo de libros tan especiales y a la vez tan olvidados. Afortunadamente, el poder aplicar sistemas de análisis científico (de una forma novedosa), tanto mediante técnicas de radiación como con técnicas físico-químicas, y el apoyarnos en un minucioso estudio histórico y visual, nos ha aportado nuevos datos hasta ahora desconocidos y ha confirmado gran parte de lo que suponíamos íbamos a encontrar tras el estudio de antiguos tratados.

Y es que los libros de coro pasaron de un obligado uso diario a quedar olvidados; esto ha llevado a que su conocimiento hoy día sea superficial, pues a pesar de que existan algunos estudios sobre los cantorales, suelen estar centrados en su catalogación o inventario como conjunto de documentos, o en el conocimiento de determinadas particularidades tales como los contenidos musicales o sus ilustraciones. Precisamente la calidad de sus miniaturas junto a esta falta de uso desde el cambio en la liturgia han hecho que se prime su valor artístico. Y nada se ha encontrado específico de los sistemas de encuadernación y protección con tapas y revestimientos; y está claro que es fundamental difundir cualquier dato que se averigüe sobre este patrimonio, insistimos, tan olvidado.

Este artículo forma parte del trabajo de investigación Los libros de coro en pergamino e ilustrados de la Abadía del Sacromonte: estudio histórico, medioambiental, de materiales y técnicas, tesis que defendí en la Facultad de Bellas Artes de la Universidad de Granada en noviembre de $2002^{2}$. 


\section{El proceso de análisis}

Al estudiar la estructura física de los libros de coro es fácil distinguir los elementos que los conforman: las cubiertas, el cuerpo y el montaje. En un estudio más detenido podremos disfrutar de los contenidos: textos (litúrgicos y musicales) e ilustraciones (miniaturas, orlas, etc.).

Sin embargo, hay varios factores que complican el análisis en profundidad, ya sea desde un punto de vista histórico como material; por ejemplo, en los libros analizados, es muy difícil determinar qué encuadernaciones son originales y cuáles han sido rehechas, o cuáles se hicieron expresamente para esta institución o se compraron o trajeron de otras ya que:

$>$ No es habitual encontrar en los libros de coro firmas que nos permitan identificar a los autores del texto, las ilustraciones o la encuadernación (que no siempre eran la misma persona), o datar fehacientemente las obras (en los analizados se han encontrado estas referencias sólo en nueve).

> Sabemos que era habitual que los encuadernadores y artistas tuvieran un taller fijo, pero también se desplazaban a las instituciones para trabajar in situ, por lo que los métodos, calidad y terminaciones de sus trabajos podían variar considerablemente.

> Cuando una institución (convento, monasterio, etc.) desaparecía, o dejaba de utilizar sus libros de coro, éstos se enviaban o vendían a otras instituciones. Este trasiego se ha dicho que fue especialmente intenso durante y después del proceso de desamortización que Mendizábal llevó hasta la iglesia con la Ley de 29 de julio de 1837, pero en el Sacromonte (y probablemente en muchas otras instituciones) y en referencia a este tipo de obras, ya ocurrió con anterioridad. Así encontramos varias noticias en los Libros de Cabildo del Sacromonte granadino referentes a este tema, como la del 3 de marzo de 1812 en la que se dice textualmente que "se acordó también se compra/sen los libros del coro que el Señor Abad y Señor Tesorero juzgasen necesarios en virtud de hallarse mui deteriorados los de Horas, Visperas, Completas y Kirial, baxo la condición de devolver los qe se compren á las Yglesias de donde se averiguase ser, siempre que por algún acontecimiento los pudiesen pedir" ${ }^{\text {. Tam- }}$ bién está datada la entrega de la Biblioteca del Convento de San Antonio de Padua el 21 de febrero de 1875 (dato que se corrobora con anotaciones en las primeras páginas de algunos cantorales). En ese trasiego también participan las donaciones, como en este caso una datada el 9 de junio de 1856 de un antiguo becario del colegio que envía desde Lisboa 5 libros de coro, que no se identifican adecuadamente en los Libros de Cabildo mencionados.

$>$ La evolución en el desarrollo del culto propició la modificación de los contenidos, por lo que algunos libros se suprimían y a otros se les incorporaban cuadernillos con nuevos textos litúrgicos (que se obtenían desmembrando otros libros o haciendo nuevos capitulos); procesos en los que a veces se recortaban los tamaños de las hojas (para adaptarlos) y/o se reducian o sustituian las tapas y revestimientos. Así en esta colección algunos han perdido los bordes (incluidos los de las orlas decorativas internas) y en algunos casos, creemos, se hicieron reencuadernaciones completas incluso al poco tiempo de su confección ${ }^{4}$.

> A veces en la institución no se solía prestar demasiado interés por datos que entonces se consideraban banales; así en los Libros de Cabildo del Sacromonte (en donde se refleja la vida diaria de la institución y que se conservan en su totalidad) no se detallan datos sobre el encargo, pago o restauración de los cantorales; y si los hay no suelen identificar las obras, los autores o los talleres en donde se encargaron.

$>$ Era y es complicado manejar estos grandes volúmenes, por lo que no era extraño su rápido deterioro; esto implicaba, naturalmente, un mantenimiento continuo que significaba en muchos casos rehacer los montajes o los sistemas de protección (cubiertas, y/o revestimientos).

> No existían criterios de intervención o restauración y estas intervenciones las hacian el propio autor, otro encuadernador o los responsables de los libros (canónigos, sochantres, etc.) con mejor o peor atino.

El estudio se complica cuando nos enfrentamos a situaciones como la siguiente: las encuadernaciones de los libros 306 (fechado en 1697), 311 (del siglo XVII), 318 (de 1619), 319 (del siglo XVII), 322 (del siglo XVII), 324 (del siglo XVII), 332 (del siglo XVII) y 333 (del siglo XVII) son iguales (en aspecto, acabado y grado de conservación) a las del libro 377, que tiene el cuerpo realizado en papel y está firmado en 1904 por el sochantre y director del coro Miguel Ramos. Esto puede suponer dos cosas: o aprovechó unas cubiertas antiguas (y por tanto ha desaparecido un volumen completo), o todas estas encuadernaciones son de finales del siglo XIX o principios del siglo XX. Por los datos recogidos, nos decantamos por la primera opción.

Sin embargo, hay elementos que nos permiten afirmar categóricamente que algunos de estos libros se hicieron expresamente para el Sacromonte granadino; dos de ellos, bastante determinantes, son las decoraciones en forma de la estrella de la Sabiduría de seis puntas (símbolo de la Abadia) que aparecen en ilustraciones y bollones; elementos en los que también se incluyen representaciones de las placas de los libros plúmbeos (origen de la aparición de esta institución), o el escudo del arzobispo fundador, Pedro de Castro Vaca (o Cabeza de Vaca) y Quiñones (Burgos 1534-Sevilla 1623).

El intentar conocer los materiales y los procesos de creación de esta tipología de libros nos ha llevado a constatar la escasez de bi- 


\section{Criterios}

La encuadernación de los libros de coro: las cubiertas de los cantorales de la Abadía del Sacromonte de Granada

\section{Javier Bueno Vargas}

bliografía que hay en castellano, tanto sobre la fabricación de soportes proteínicos (pergamino y cuero), como de las técnicas de encuadernación o de ilustración de los cantorales, o sobre la conservación o restauración de estas obras. Tampoco es fácil hacer un seguimiento histórico a causa de las importantes lagunas documentales y por la inexistencia de estudios generalizados y actualizados de las colecciones de libros de coro que permitan al menos hacer estudios comparativos.

\section{La encuadernación de libros de coro}

Según la definición de la Real Academia Española, encuadernar es "juntar, unir y coser varios pliegos o cuadernillos y ponerles cubiertas" ${ }^{\prime \prime}$. Recordemos que esta forma de presentar los textos se remonta al siglo IV d.C., cuando se pasó del rollo a las hojas lisas plegadas.

Básicamente, estas encuadernaciones se pueden analizar, como vamos a ver, desde dos puntos de vista: el meramente funcional, como sistema de protección de los contenidos, y artístico, puesto que en nuestro caso, como en otros muchos, se pretende además que esta protección sea agradable, estética. Por ello los cueros que revisten las tapas se policroman o se gofran y los bollones, esquineras o cierres se hacen con distintos materiales, formas y decoraciones.

En general el proceso seguido en la creación de los cantorales y, en este caso, los del Sacromonte, es homogéneo:

1. Se crean bifolios, uniendo dos pieles convertidas en pergamino.

2. Luego se agrupan formando cuadernillos: biniones, terniones, cuaterniones (de dos, seis u ocho folios respectivamente) que se numeran, escribiéndose los textos y realizándose las ilustraciones.

3. El cuerpo se forma ordenando, agrupando y cosiendo los cuadernillos con hilos de lino o cáñamo (bramante) a nervios de cuero o de cuerda (sencillos o dobles).

4. Las tapas se hacen mediante ensamblaje de tablas de madera que luego se forran con cueros que, además, se tensan mediante clavos en los tres cantos interiores de la tapa, (el superior, delantero e inferior); también se pegan al plano con adhesivos orgánicos (engrudo o colas animales).

5. La unión del cuerpo a las tapas se consigue pasando los nervios a través de perforaciones en la madera, en las que se clavan o embuten y que en ocasiones se sellan con injertos también de madera.

6. Para disimular esta unión, internamente se colocan las guardas sencillas, pegando y/o clavando palimpsestos (hojas de pergamino pertenecientes a otros cantorales), papel (tanto blanco como de
Tabla 1. Características generales de los cantorales analizados con radiografía Figura 1. Radiografias y esquemas de los libros 318, 316, 312 y 303

hojas de manuscritos reciclados), o cartones (en nuestro caso colocados en restauraciones ya que el encontrado es el mismo en libros de diferentes épocas). En todos los casos, los elementos se adhieren con cola animal y/o se clavan con puntas de hierro o bronce.

7. El lomo se hace con otra piel y en estos libros es recto y hueco y está reforzado internamente con una tela pegada.

8. En ocasiones se colocaban cierres (dos en los libros analizados).

9. El proceso en estos libros acaba con el clavado de una chapa doblada por todos los cantos de las tapas y en la zona de unión de ésta al lomo, con infinidad de puntas (se han contabilizado hasta más de 250 por tapa).

10. La protección se podía incrementar con el clavado de bollones y esquineras (elementos metálicos que además se trabajaban para que decorasen) y que no siempre están presentes.

Vamos a ver detenidamente los elementos que conforman las cubiertas.

\section{Características de las cubiertas de los libros de coro}

El gran tamaño y peso de los cantorales, características únicas de esta tipología, les llevan a necesitar de un sistema de encuadernación muy resistente para el que se utilizan ciertos materiales inusuales en otros libros. Las dimensiones de los volúmenes de esta colección varían desde los más pequeños, de tamaño folio, hasta la medida media de estos libros, que ronda el medio metro de ancho por unos $80 \mathrm{~cm}$ de alto. El más grande es el número 318 con $650 \mathrm{~mm}$ de ancho por 920 de alto, $100 \mathrm{~mm}$ de grosor medio y un peso de $30 \mathrm{Kg}$.

Por ello, la estructura de los libros de coro es la tradicional de cualquier libro, con algunas particularidades en el caso de las tapas:

> Están confeccionadas con tablas de madera de unos 15-20 mm de grosor, ensambladas entre sí de diferentes formas como se ha podido ver con las radiografías realizadas.

> Están reforzadas en su perímetro, como ya se ha mencionado, con chapas de cinc.

$>$ El revestimiento suele ser cuero liso y brillante, aunque a veces es piel vuelta. En algunos casos la piel está gofrada (los libros 301 a 305, 321, 336, 338, 327 y 378), es decir, decorada con hierros candentes que presionados sobre la piel la queman dejando un surco ennegrecido con diversos dibujos. 


\begin{tabular}{|c|c|c|c|c|c|c|c|c|c|}
\hline $\begin{array}{l}\text { № } \\
\text { libro }\end{array}$ & $\begin{array}{l}\text { № } \\
\text { original }\end{array}$ & $\begin{array}{l}\text { Estado de } \\
\text { conservación } \\
\text { general }\end{array}$ & $\begin{array}{l}\text { Ancho } \\
\text { total (mm) }\end{array}$ & $\begin{array}{l}\text { Alto } \\
\text { total } \\
(\mathrm{mm})\end{array}$ & $\begin{array}{l}\text { Grosor } \\
\text { medio } \\
(\mathrm{mm})\end{array}$ & $\begin{array}{l}\text { Peso } \\
\text { total } \\
(\mathrm{Kg})\end{array}$ & Autor texto & $\begin{array}{l}\text { Fecha de } \\
\text { realización }\end{array}$ & $\begin{array}{l}\text { Lugar de } \\
\text { realización }\end{array}$ \\
\hline 303 & $6 ?$ & Muy malo & 530 & 800 & 105 mm & 20 kg & F.D.Q. Ordinis Praedicat & 1656 a II agosto 1657 & Córdoba? \\
\hline 312 & 14 & Muy malo & 615 & 795 & 103 mm & $20 \mathrm{Kg}$ & Desconocido & $X V I I$ & Granada? \\
\hline 316 & 17 & Muy malo & 600 & 830 & $130 \mathrm{~mm}$ & $23 \mathrm{Kg}$ & El mismo del libro 372 & $X V I I$ & Granada? \\
\hline 318 & 19 & Regular & 650 & 920 & 100 mm & 30 kg & Pedro Marañón & 1619 & Málaga \\
\hline
\end{tabular}

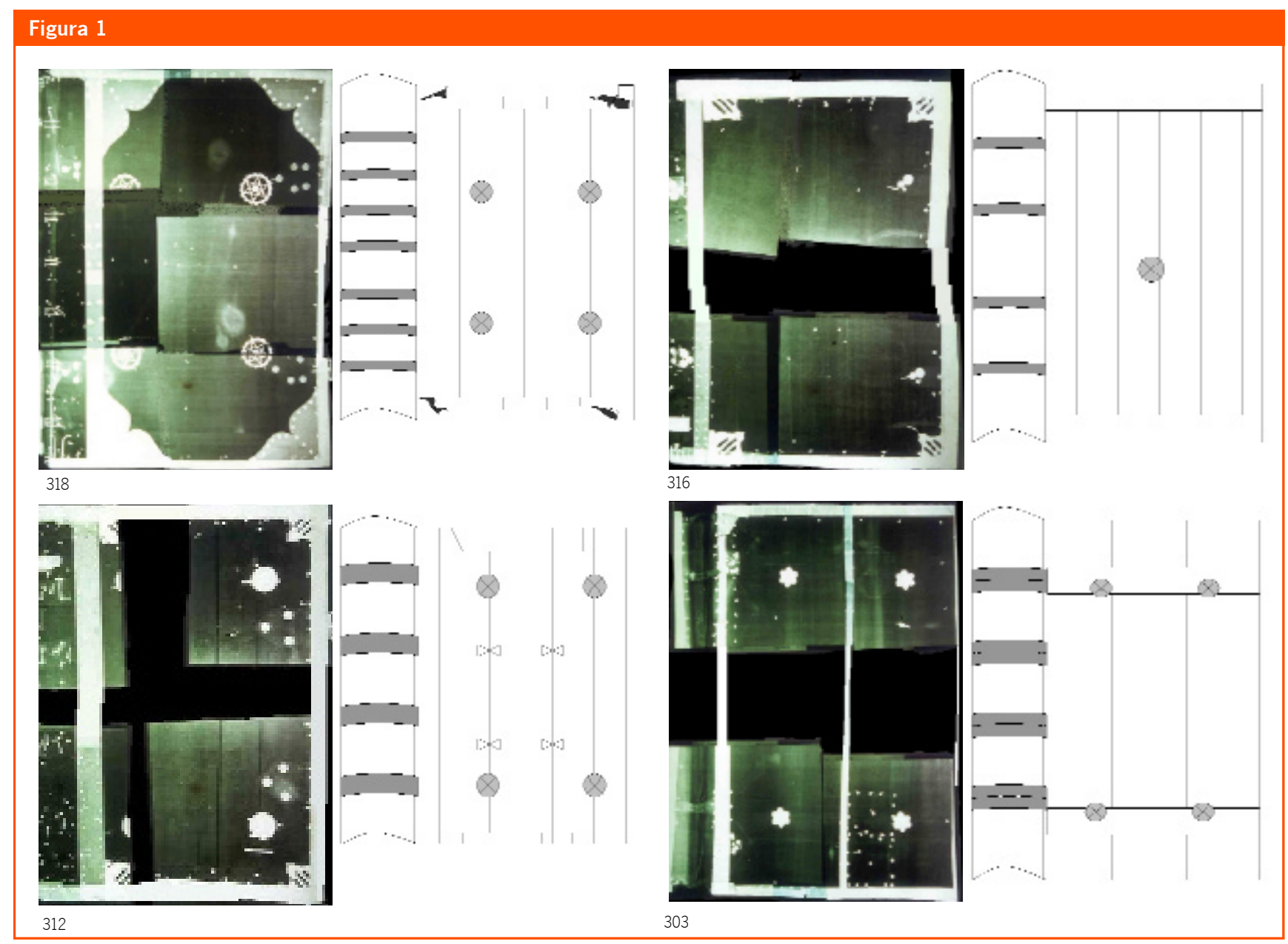

> Los elementos decorativos son muy sobrios: bollones y esquineras, que además protegen al libro de golpes y rozaduras y que destacan por su volumen (15 mm de media).

> Los sistemas de cierre en estos libros pueden ser de dos tipos: hebillas que sujetan tiras de cuero agujereada (a modo de cinturón), o broches, con dos piezas metálicas que se engarzan entre sí. En esta colección, si los hay, son de hebilla.

> Las dos tiras de cuero que se clavan al lomo y que sirven para sacar el volumen de la estantería son también elementos específicos de los cantorales.

\section{Las tapas}

Ya desde la Edad Media se solian hacer de madera, sobre todo en los libros de gran formato, como éstos. En Francia e Inglate- rra se usaba habitualmente el roble y el pino y el haya en Italia (lo que hacía más ligeros los volúmenes) ${ }^{6}$. En general, todos los libros analizados conservan los revestimientos de las tapas, la cubierta de cuero exterior y las guardas interiormente, por lo que se ha recurrido a las radiografías para llegar a conocer el número de piezas, los sistemas de ensamblaje y el estado de las tablas que se usaron?

En las radiografias de las tapas, vemos la cantidad de clavos que se podian llegar a emplear para sujetar los nervios a las maderas, especialmente en el caso del libro 312.

La estructura de las tapas

Se han seleccionado cuatro tipologías diferentes en función de los acabados de las cubiertas y los tipos de ilustraciones que contienen; son los libros 303, 312, 316 y 318. Han sido las que se han creído representativas del resto de los volúmenes y cuyos datos generales básicos los recogemos en la tabla 1. 


\section{Criterios}

La encuadernación de los

libros de coro: las

cubiertas de los

cantorales de la Abadía

del Sacromonte de

Granada

Javier Bueno Vargas
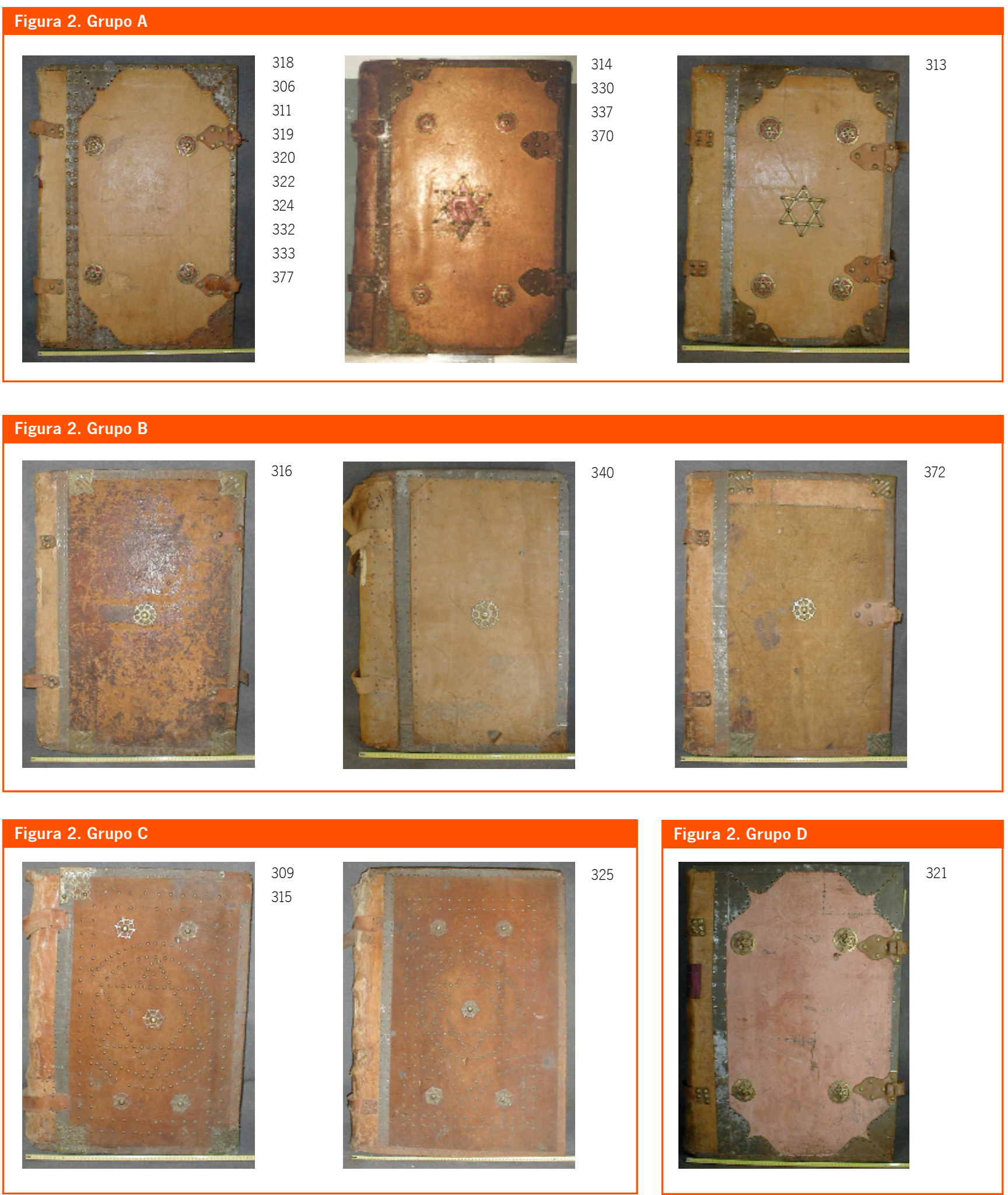

Figura 2. Grupo D

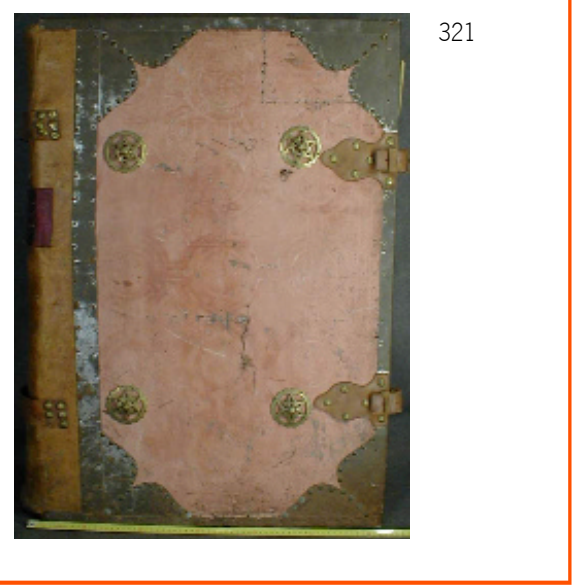



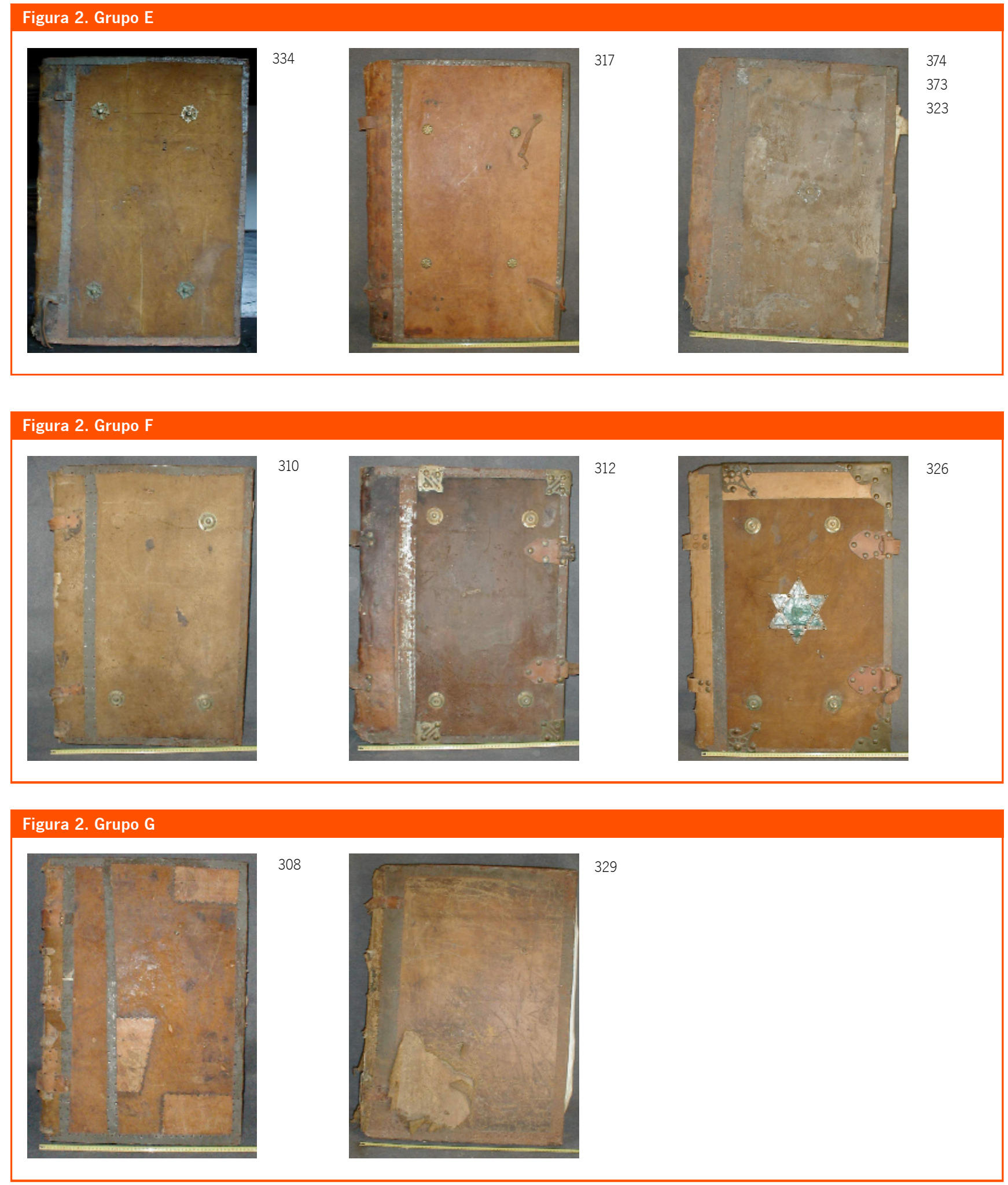


\section{Criterios}

La encuadernación de los libros de coro: las cubiertas de los cantorales de la Abadía del Sacromonte de Granada

\section{Javier Bueno Vargas}

Figura 2. Grupo H

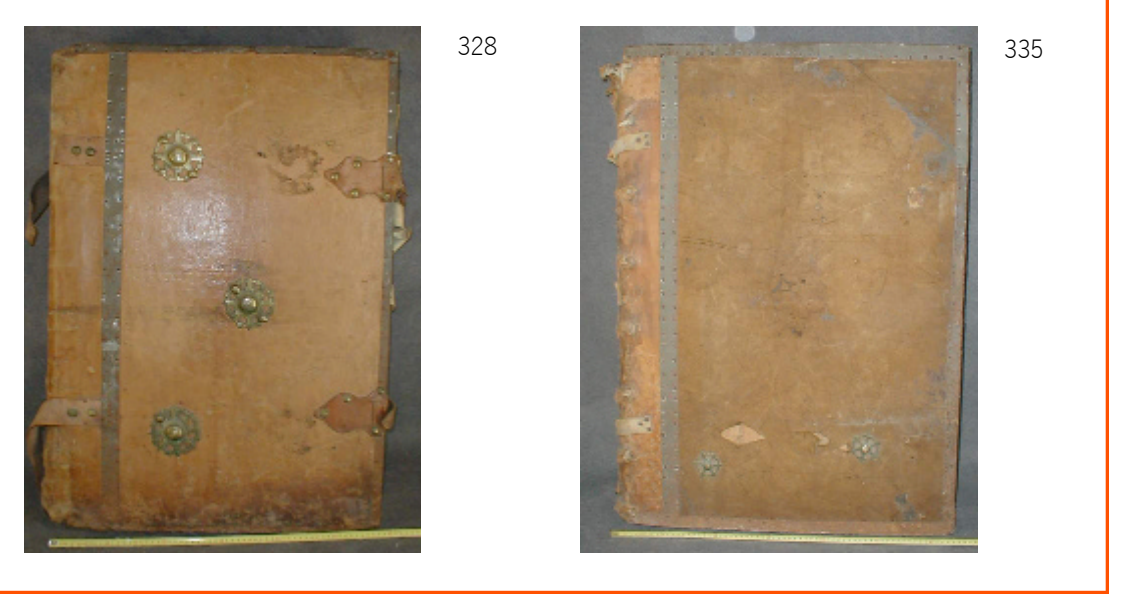

\section{Figura 2. Grupo I}

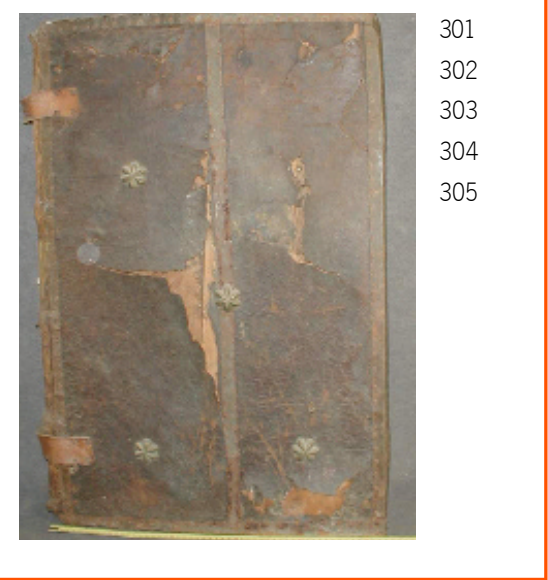

Figura 2. Grupo J
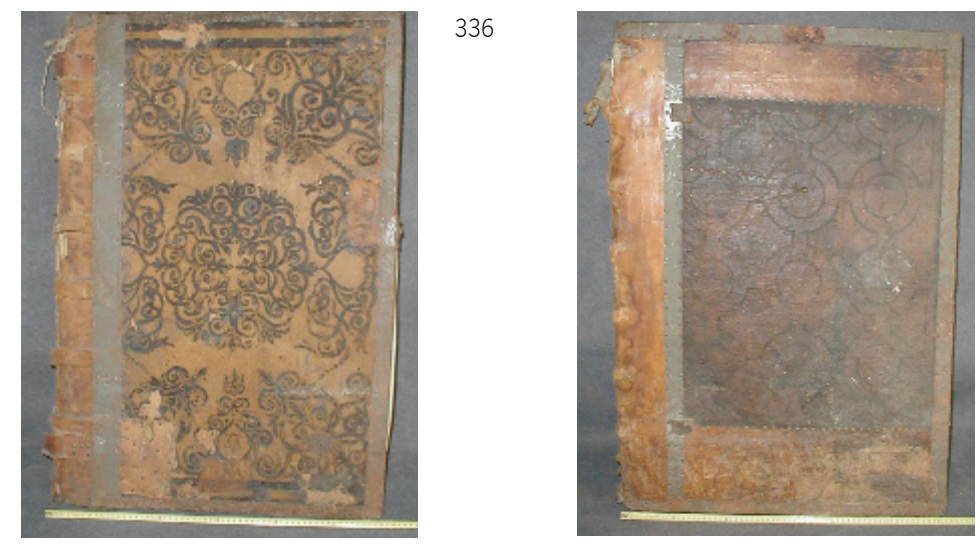

338 327

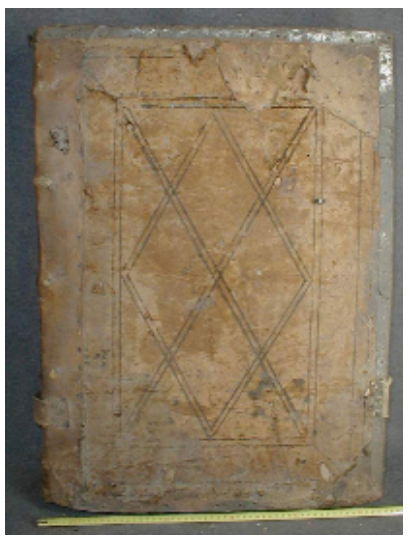

378

Las radiografías se han interpretado en esquemas (Fig. 1). En ellas y, en general, se han detectado listones obtenidos mediante cortes longitudinales (tangenciales, radiales o axiales), en tron$\cos ^{8}$ de pino o haya (en las tapas radiografiadas). La estructura tabular se conforma generalmente con pocas piezas, entre 3 y 6 listones verticales, que se refuerzan con otros listones perimetralmente o perpendicularmente en la mitad del plano; en el caso de la tipología representada por el libro 312, Ios listones van unidos con piezas en cola de milano.

Como característica común destaca que no se haya detectado ningún elemento metálico para la unión entre maderas, así que ésta se hace mediante encolado con uniones vivas, a media madera o con sistemas de refuerzos internos (como espigas de madera). También lo es el hecho de que estas uniones son muy exactas entre los listones de mayor tamaño y sólo hay desajustes y huecos en las esquinas, sobre todo en las situadas junto al lomo del libro, en donde se acumulan pequeñas piezas de diversas formas y tamaños con formas caprichosas.

Respecto a la calidad de las maderas y aunque se han encontrado nudos de gran tamaño en algunos casos, predominan las tablas seleccionadas, homogéneas en su estructura (tipo de veta y corte elegido). Están muy bien curadas por lo que son muy estables y no hay deformaciones como alabeamientos (combamientos) o desplazamientos de piezas.

No se han detectado grietas o fendas salvo en aquellos cantorales que han estado en ambientes inadecuados y que son los que están en peor estado en general; es el caso del libro 336 en el 


\begin{tabular}{|lll|}
\hline Tabla 2 & & \\
\hline № registro del libro & $№$ clavostapa anterior / perdidos de unión de la chapa & № clavos tapa posterior / perdidos de unión de la chapa \\
300 & $40 / 1$ & $41 / 0$ \\
302 & $208 / 14$ & $211 / 5$ \\
323 & $226 / 15$ & $243 / 13$ \\
329 & $240 / 5$ & $272 / 5$ \\
334 & $231 / 17$ & $203 / 18$ \\
335 & $190 / 6$ & $206 / 5$ \\
\hline
\end{tabular}

que la separación de las tablas ha fracturado también el revestimiento de cuero. Aunque en las radiografias realizadas no se ha detectado ataque de insectos xilófagos, si lo ha habido en la mayoría de los libros (ataque ya finalizado) y han llegado a atravesar los cueros de las cubiertas o las guardas para salir al exterior. Su presencia no ha afectado al cuerpo de los libros analizados, quizá por la alcalinidad de los pergaminos.

\section{Las cubiertas o revestimientos}

Habitualmente el exterior de la tapa se recubría con piel curtida o vuelta que algunas veces se coloreaba; desde mediados del siglo XIV, se generaliza el decorarla con impresiones de flores, animales y dibujos geométricos, a veces mediante gofrado ${ }^{9}$. Aunque esta piel se puede pegar a la madera con distintos tipos de adhesivos, los más utilizados han sido las colas proteínicas (cola de conejo o de carpintero), el engrudo de harina y la cola de almidón. En los libros analizados, por los restos de cola visibles y el grado de cristalización detectado, sabemos que se han utilizado colas proteínicas.

\section{El cuero}

Microscópicamente ${ }^{10}$ la piel viva es una complicada colección de moléculas de proteinas fibrilares, (principalmente colágeno ${ }^{11}$ además de elastina y reticulina, junto a la grasa animal y los taninos libres), así como una gran cantidad de agua. Estas fibras están entrelazadas entre sí en largas cadenas para formar una heterogénea estructura básica, a pesar de que las complejas moléculas de colágeno tiendan a orientarse en la misma dirección. Precisamente las partes terminales de las cadenas causan que la piel sea muy vulnerable a los agentes que la desintegran (biológicos o climáticos) por lo que deben ser protegidas para hacer al derivado obtenido de ella (cuero, pergamino, etc.) un material más duradero.

La piel tratada para convertirse en cuero está constituida mayoritariamente por colágeno proveniente de la dermis (elemento que en peso es el 90\%). La aplicación de tratamientos y manipulaciones de la piel, a la vez que mejoran algunas de sus características (cualidades mecánicas, suavidad, flexibilidad y sobre todo su resistencia biológica y al medioambiente), nos permiten su estabilización. En el caso del cuero, el sistema más frecuente de estabilización es mediante la aplicación de taninos (básicamente en tres procesos denominados al cromo, vegetal y al ácido ${ }^{12}$ ). Los taninos, que bloquean y hacen insolubles las terminaciones de las moléculas de colágeno entrecruzando los encadenamientos de las largas moléculas, pueden ser inorgánicos, orgánicos y sintéticos ${ }^{13}$; eran ya conocidos por egipcios y mesopotámicos (que utilizaban los orgánicos de origen vegetal). Para estabilizar la piel también se usan otros procesos, como los que usan aceite o alumbre (sulfato de aluminio y potasio).

El proceso de fabricación del cuero es el siguiente ${ }^{14}$.

1. Despellejado y separación de la carne y salado para su traslado a la fábrica en donde se limpia y se sala de nuevo si no va a ser tratada inmediatamente. La sal debe ser eliminada completamente.

2. Separación de las capas pilosa (y consecuente eliminación del pelo) y de la capa carnosa; se consigue mediante tratamiento enzimático (con enzimas queratinolíticas o depilantes ${ }^{15}$ ) que modifican químicamente su composición. Se hace en baños que se remueven lentamente de 48 a 72 horas.

3. Purificación y eliminación mecánica del pelo, mediante lavado alcalino que se puede alternar con los primeros estiramientos que se hacen manual o mecánicamente.

4. Eliminación de la alcalinidad y preparación para recibir los curtientes, lo que se puede conseguir con enzimas especiales, en un proceso que se llama rendido; se aplican posteriormente ácidos (como el fórmico, sulfúrico o acético), que acaban con la actividad enzimática (es el proceso que se llama piquelado).

5. Curado y estabilización, que se consigue mediante la aplicación fundamentalmente de taninos.

6. Acabado, que se consigue alternando el estirado con el teñido y/o aplicación de grasas líquidas para aportarle o modificar el color, la suavidad, la flexibilidad, la resistencia o la textura. 


\section{Criterios}

La encuadernación de los libros de coro: las cubiertas de los cantorales de la Abadía del Sacromonte de Granada

\section{Javier Bueno Vargas}

Figura. 3. Evolución de la esquinera para convertirse en elemento decorativo. Libros 330,300 y 306

Figura.4. Tipologías de los bollones de los libros del Sacromonte
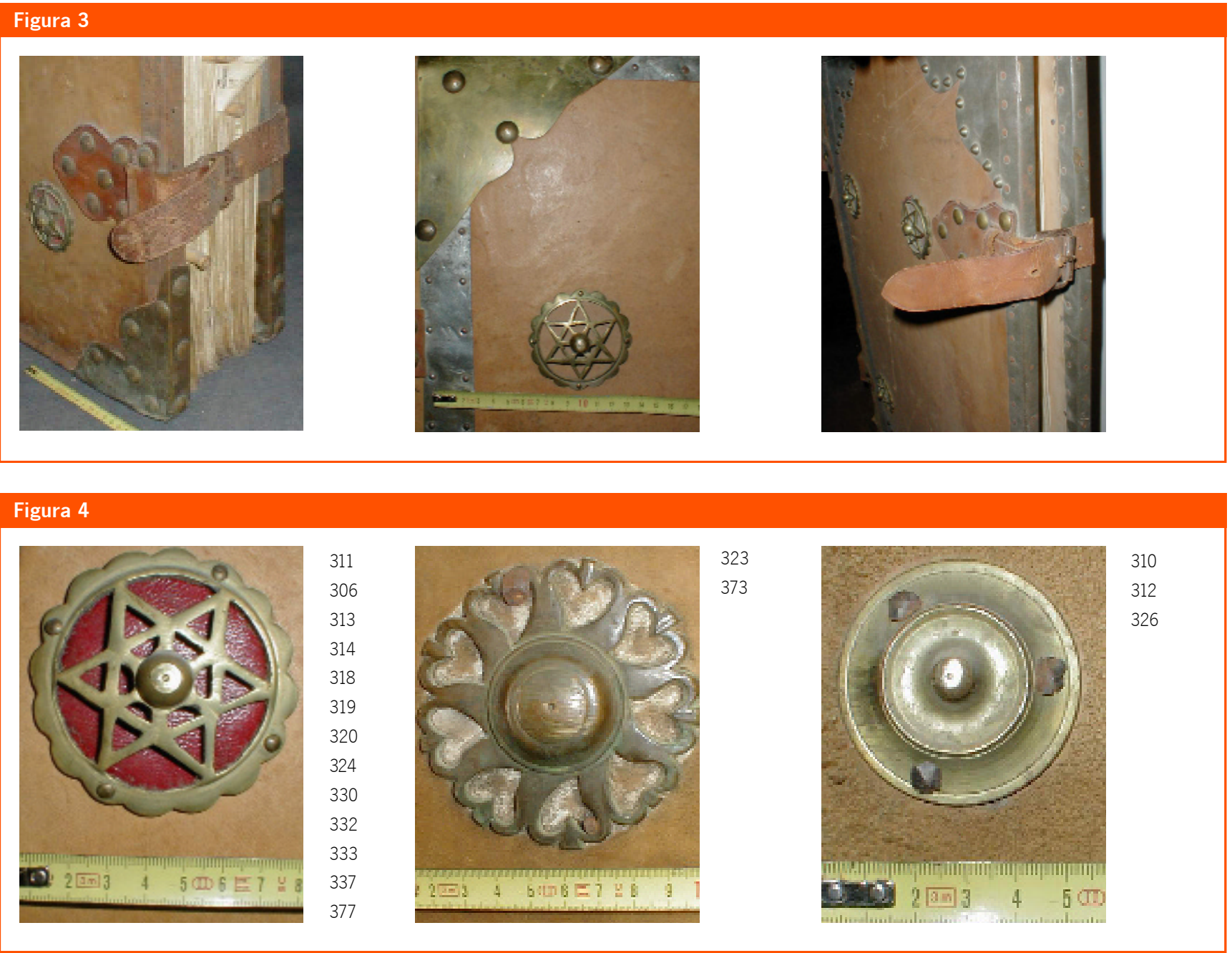

7. Secado al aire ya que todos estos procesos son en húmedo o baño; a diferencia del pergamino, durante el secado no es necesario mantener la piel tensada en un telar.

8. En ocasiones se aplica un acabado. Actualmente consiste simplemente en su pulido, en una máquina de abrillantar, pero también se les puede aplicar una capa final de pigmentos que van en una suspensión de barnices de resinas termoplásticas y con los que se le puede aportar mayor brillo, color, rigidez, textura o impermeabilidad.

9. El gofrado (por rubefacción o tostado mediante hierros calentados) y la aplicación de ceras para aumentar el brillo se suelen hacer una vez montados los revestimientos.

Basándonos fundamentalmente en las tipologías de los elementos decorativos y el acabado del plano se ha realizado la Fig. 2, que consiste en una clasificación de los revestimientos de estos libros en 10 grupos, cuyas características son las siguientes:

Grupo A: La piel es cuero de acabado liso y brillante de color ocre. Los elementos decorativos son 4 bollones en bronce con forma de estrella de seis puntas sobre fondo rojo y en el caso de los libros 314, 330, 337 y 370 hay una estrella de características semejantes a los bollones (con menos grosor) en el centro de cada plano; en el libro 313 ésta no tiene el fondo rojo. Todas tienen esquineras (reales o simplemente decorativas).

Grupo B: La piel es chagrín, piel de Nigeria o piel de Levante. Piel sobre todo de cabra cuya superficie tiene un acabado texturado mediante granulado o con pequeñas arrugas homogéneas; la encontramos en los libros 316, 340 y 372 . Sólo en el primero hay esquineras teniendo los tres en común el tipo de bollón del centro. 


\begin{tabular}{|c|c|c|c|c|c|c|c|}
\hline $\begin{array}{l}\text { № } \\
\text { libro }\end{array}$ & $\begin{array}{l}\text { Estado de } \\
\text { conservación }\end{array}$ & $\begin{array}{l}\text { № piezas } \\
\text { tenia/conserva }\end{array}$ & Diámetro & $\begin{array}{l}\text { Grosor } \\
\text { máximo }\end{array}$ & $\begin{array}{l}\text { Unión de la ornamentación } \\
\text { a la tapa }\end{array}$ & Oxidación & $\begin{array}{l}\text { Pérdida de clavos } \\
\text { de unión }\end{array}$ \\
\hline 300 & muy bueno & $8 / 7$ & $70 \mathrm{~mm}$ & $12 \mathrm{~mm}$ & clavada con clavos de bronce & ninguna & si en la pieza perdida \\
\hline 301 & bueno & $10 / 9$ & $40 \mathrm{~mm}$ & $8 \mathrm{~mm}$ & clavada con clavos de bronce & no tiene & no tiene \\
\hline 302 & malo & $10 / 4$ & $40 \mathrm{~mm}$ & $10 \mathrm{~mm}$ & clavada con clavo de bronce & todas & si en las 4 piezas perdidas \\
\hline 303 & aceptable & $10 / 10$ & $40 \mathrm{~mm}$ & $8 \mathrm{~mm}$ & clavada con clavos de bronce no visibles & todas, pero en grado inicial & si \\
\hline 304 & aceptable & $10 / 6$ & $40 \mathrm{~mm}$ & $10 \mathrm{~mm}$ & clavada con clavos de bronce & todas & no tiene \\
\hline 305 & muy bueno & $10 / 9$ & $40 \mathrm{~mm}$ & $10 \mathrm{~mm}$ & clavada con clavos de bronce & todas & no tiene \\
\hline 306 & muy bueno & $8 / 8$ & $75 \mathrm{~mm}$ & $15 \mathrm{~mm}$ & clavada con clavos de bronce & no tiene & no tiene \\
\hline 308 & no tiene & no tiene & no tiene & no tiene & no tiene & no tiene & no tiene \\
\hline 309 & aceptable & $10 / 10$ & $50 \mathrm{~mm}$ & $8 \mathrm{~mm}$ & clavada con clavos de bronce & todas & no tiene \\
\hline 310 & malo & $8 / 7$ & $50 \mathrm{~mm}$ & $15 \mathrm{~mm}$ & clavada con clavos de bronce & todas & 4 \\
\hline 311 & regular & $8 / 8$ & $50 \mathrm{~mm}$ & $15 \mathrm{~mm}$ & clavada con clavos de bronce & ninguna & no \\
\hline 312 & malo & $8 / 8$ & $30 \mathrm{~mm}$ & $6 \mathrm{~mm}$ & clavada con clavos de bronce & no tiene & no tiene \\
\hline 313 & bueno & $10 / 10$ & $75 \mathrm{~mm}$ & $15 \mathrm{~mm}$ & clavada con clavos de bronce & no tiene & no tiene \\
\hline 314 & aceptable & $10 / 10$ & $75 \mathrm{~mm}$ & $15 \mathrm{~mm}$ & clavada con clavos de bronce & todas & no tiene \\
\hline 315 & muy bueno & Ver grupo C & $30-5 \mathrm{~mm}$ & $5 \mathrm{~mm}$ & clavada con clavos de bronce & todas & no tiene \\
\hline 316 & aceptable & $2 / 2$ & $70 \mathrm{~mm}$ & $18 \mathrm{~mm}$ & clavada con clavos de hierro & puntualmente & no \\
\hline 317 & muy bueno & $8 / 8$ & $30 \mathrm{~mm}$ & $4 \mathrm{~mm}$ & clavada con clavos de bronce & dos & no tiene \\
\hline 318 & aceptable & $8 / 8$ & $70 \mathrm{~mm}$ & $20 \mathrm{~mm}$ & clavada con clavos de bronce & todas & no \\
\hline 319 & aceptable & $8 / 8$ & $70 \mathrm{~mm}$ & $15 \mathrm{~mm}$ & clavada con clavos de bronce & dos & no tiene \\
\hline 320 & bueno & $8 / 8$ & $75 \mathrm{~mm}$ & $15 \mathrm{~mm}$ & clavada con clavos de bronce & dos & no tiene \\
\hline 321 & muy bueno & $10 / 8$ & $70 \mathrm{~mm}$ & $15 \mathrm{~mm}$ & clavada con clavos de bronce & ninguna & no tiene \\
\hline 322 & muy bueno & $8 / 8$ & $70 \mathrm{~mm}$ & $15 \mathrm{~mm}$ & clavada con clavos de bronce & todas & no tiene \\
\hline 323 & muy malo & $10 / 6$ & $40 / 440 \mathrm{~mm}$ & $10 \mathrm{~mm}$ & la central clavada con clavos de hierro & todas & no \\
\hline 324 & muy bueno & $8 / 8$ & $70 \mathrm{~mm}$ & $15 \mathrm{~mm}$ & clavada con clavos de bronce & todas & no tiene \\
\hline 325 & aceptable & $10 / 10$ & $55 \mathrm{~mm}$ & $15 \mathrm{~mm}$ & clavada con clavos de bronce & todas & no tiene \\
\hline 326 & muy bueno & $14 / 13$ & $50 \mathrm{~mm}$ & $10 \mathrm{~mm}$ & clavada con clavos de bronce & dos en tapa posterior & no tiene \\
\hline 327 & no tiene & $10 ? / 0$ & $120 \mathrm{~mm}$ & & clavada con clavos de bronce & no tiene & todos \\
\hline 328 & regular & $10 / 8$ & $90 \mathrm{~mm}$ & $15 \mathrm{~mm}$ & clavada con clavos de bronce & ligeramente & 13 \\
\hline 329 & no tiene & no tiene & no tiene & no tiene & no tiene & no tiene & no tiene \\
\hline 330 & regular & $10 / 10$ & $70 \mathrm{~mm}$ & 20 mm & clavada con clavos de bronce & todas & no tiene \\
\hline 332 & aceptable & $8 / 8$ & $70 \mathrm{~mm}$ & $15 \mathrm{~mm}$ & clavada con clavos de bronce & ligeramente & no tiene \\
\hline 333 & bueno & $8 / 8$ & $75 \mathrm{~mm}$ & $15 \mathrm{~mm}$ & clavada con clavos de bronce & ligeramente & no tiene \\
\hline 334 & malo & $10 / 5$ & $60 \mathrm{~mm}$ & $10 \mathrm{~mm}$ & clavada con 1 clavo de bronce & todas & todos \\
\hline 335 & muy malo & $10 / 2$ & $60 \mathrm{~mm}$ & $10 \mathrm{~mm}$ & clavada con 1 clavo de bronce & todas & faltan 8 piezas \\
\hline 336 & no tiene & no tiene & no tiene & no tiene & no tiene & no tiene & no tiene \\
\hline 337 & bueno & $10 / 10$ & $70 \mathrm{~mm}$ & $15 \mathrm{~mm}$ & clavada con clavos de bronce & no tiene & 4 \\
\hline 338 & no tiene & no tiene & no tiene & no tiene & clavada con clavos de bronce & no tiene & no tiene \\
\hline 340 & bueno & $2 / 2$ & $75 \mathrm{~mm}$ & $10 \mathrm{~mm}$ & clavada con clavos de bronce & no tiene & no tiene \\
\hline 341 & bueno & $10 / 9$ & $50 \mathrm{~mm}$ & $18 \mathrm{~mm}$ & clavada con clavos de bronce & todas & no tiene \\
\hline 369 & malo & $8 / 8$ & $70 \mathrm{~mm}$ & & clavada con clavos de hierro & todas & 1 \\
\hline 370 & pérdidas & $2 / 0$ & $140 \mathrm{~mm}$ & & clavada con clavos & & todos \\
\hline 372 & malo & 2 & $70 \mathrm{~mm}$ & $10 \mathrm{~mm}$ & clavada con clavos de bronce & ninguna & no tiene \\
\hline 373 & bueno & $10 / 10$ & 80 y 100 mm & $10 \mathrm{~mm}$ & clavada con clavos de bronce & ligeramente & no \\
\hline 374 & bueno & $2 / 2$ & $60 \mathrm{~mm}$ & $8 \mathrm{~mm}$ & clavada con clavos de bronce & no tiene & no tiene \\
\hline 378 & no tiene & $10 / 10$ & $80 \mathrm{~mm}$ & & clavada con clavos de bronce & $\begin{array}{l}\text { todas, han dejado huella } \\
\text { de óxido en la piel }\end{array}$ & todos \\
\hline
\end{tabular}




\section{Criterios}

La encuadernación de los libros de coro: las cubiertas de los cantorales de la Abadía del Sacromonte de Granada

\section{Javier Bueno Vargas}

Grupo C: Este grupo se caracteriza por contar además de con cinco bollones, con multitud de chinchetas de bronce con las que se realiza una estrella central y se compartimenta el resto de la superficie de cada tapa con elementos geométricos.

Grupo D: Está formado por un solo libro, el 321; se caracteriza por ser el único revestimiento con estrato de color (rosa claro). Debajo se aprecian restos de gofrado.

Grupo E: Se trata de revestimientos de piel vuelta bastante deteriorados cuyo nexo de unión es también el tipo de bollón.

Grupo F: Se han agrupado con el mismo criterio que el grupo anterior.

Grupo G: Los libros de este grupo son los de revestimientos más sencillos pues no tienen elementos decorativos.

Grupo H: Los libros 328 y 335 tienen bollones con calado en forma de corazones que conforman círculos.

Grupo I: En este grupo se han reunido los revestimientos de piel lisa y brillante gofrados con cuadrados creados con cenefas de círculos y triángulos y con motivos vegetales en las esquinas interiores y centros de cada uno.

Grupo J: También los de este grupo están gofrados con diferentes motivos más sencillos que los del grupo anterior.

\section{Los elementos accesorios metálicos}

Podemos encontrarnos estos elementos como sistemas de unión de piezas y conformando distintos elementos (esquineras y bollones).

\section{Elementos metálicos de sujeción}

Se han utilizado fundamentalmente para la unión de la chapa que rodea la mayoría de las tapas y también de los nervios, boIlones y esquineras; en la Fig. 1 vemos parte de los empleados en la tapa del libro 312 y se han llegado a contabilizar más de 500 puntillas por tapa. Sirvan como ejemplo los que mostramos en la tabla 2.

Se ha escogido el caso del libro 300 porque se sale de la norma (sólo presenta 82 clavos); lo habitual es encontrar, como se ve en los ejemplos de la tabla anterior, unos cuatrocientos clavos sujetando las chapas que rodean el canto de los libros (+/- 200 por tapa); incluso pueden llegar a ser más de 500 (como en el caso del libro 329 o si contamos además los clavos empleados en las chapas que refuerzan la unión del lomo a las tapas). La mayoría son puntas de hierro de entre 15 y $20 \mathrm{~mm}$ de longitud y de 1 a $2 \mathrm{~mm}$ de diámetro, que están oxidadas en la casi totalidad de los casos; hay algunos ejemplares en los que se han utilizado tachuela de bronce. Además se han empleado, en menor medida, para sujetar y/o reforzar la unión de pergaminos o cartones que forran el interior de las tapas, generalmente en labores de restauración mal ejecutadas.

Hay otros elementos metálicos que son pequeñas arandelas clavadas en la mitad de la tapa, una en cada cara; en ellas se enganchaban las cadena que mantenía al libro sujeto y abierto en el facistol. En la mayor parte se han perdido, pero quedan los orificios de haberlas tenido.

\section{Esquineras y bollones}

Los otros elementos metálicos que encontramos son las esquineras y bollones, que también reciben el nombre de clavería ${ }^{16}$.

Ambos elementos surgen como remedio al deterioro debido a la manipulación de estos grandes y pesados libros. Con las primeras se evitaba el astillamiento de las esquinas y, con los segundos, el arañazo y roce de las pieles de las cubiertas ${ }^{17}$. Respecto a los autores o los precios pagados, decir que no se han encontrado referencias directas en los archivos de la Abadia ${ }^{18}$.

De las esquineras vemos que ha habido una evolución desde el momento en que se emplearon hasta que se convirtieron en meros elementos simulados. Lo vemos en la secuencia de la Fig. 3. En la de la izquierda, vemos que la esquinera es un elemento útil; realizada en bronce, rodea y protege la esquina. En la del centro, ya es una chapa de bronce que, aunque rebordea el canto, no lo protege ya que éste se refuerza realmente con una chapa de hojalata. En la de la derecha, la esquinera se ha convertido en un mero adorno que no cumple ninguna función salvo la decorativa ya que el borde y esquina se refuerzan con una chapa clavada.

En referencia a los bollones o botones, recordemos que son aquellos elementos metálicos que están presentes en la tapa y la protegen de golpes. Los de los cantorales del Sacromonte son de diversas tipologías, aunque todos están confeccionados en bronce; son chapas de unos milimetros que se ahuecan y recortan en forma semiesférica para conseguir un volumen que oscila entre los 5 y $20 \mathrm{~mm}$; son siempre circulares. Recogemos sus características más destacadas en la tabla 3.

Algunos llevan fondos de piel de color rojo (grupo 1) y otros restos de pintura blanca (libros 323 y 373). Podemos agrupar los tipos de bollones, por similitud estética, en distintas categorías (Fig. 4). Los bollones más abundantes son los que tienen forma de estrella (Fig. 4a). 
Notas

${ }^{1}$ Los que se identifican con los números 300 al 341 y del 369 al 378 y se han analizado excluyendo los numerados con el 331, 339 y 376, que no se han localizado. La numeración se corresponde con la empleada en el último inventario de bienes que se hizo en la Abadía del Sacromonte

${ }^{2}$ Esta tesis fue dirigida por la doctora Teresa Espejo Arias y valorada con sobresaliente cum laude por unanimidad

${ }^{3}$ Destacamos otra del 26 de septiembre de 1823 del Cabildo en que se trata "la devolución de los libros de coro del Real Monasterio de San Gerónimo que de orden de su llustrísima en el tiempo de la dominación revolucionaria, y por la extinción de los monjes, fueron entrega/dos a este cavildo, y los que se mandaron devolver a los indicados Gerónimos ya restablecidos por el gobierno Real"

${ }^{4}$ Como ocurrió con la del libro 312, en el que originalmente se cosían los cuadernillos con nervios sencillos (de los que quedan marcas como los agujeros y algunas deformaciones) y luego se reencuadernaban con cintas de cuero practicando nuevos orificios

${ }^{5}$ Para más información sobre las técnicas de encuadernación se pueden consultar por ejemplo: DE CUSA, Juan. Cómo encuadernar un libro. Barcelona: Ediciones CEAC, 1996 JOHNSON, A.W. Manual de encuadernación. Madrid: Tursen-Hermann Blume Ediciones, 1993; RIBERHOLT, K. y DRASTRUP, A. Técnicas de encuadernación. Sevilla: PROGENSA SA, 1989

${ }^{6}$ DE HAMER, Christopher. Medieval craftsmen: scribes and illuminators. Londres: British Museum Press, 1995, p. 67

${ }^{7}$ Para profundizar más en este tema son interesantes, por ejemplo, MIÑARO LÓPEZ, J.M. La preparación de la madera: el embón, actas del Primer Simposium Nacional de Imaginería, Se villa: Colegio Oficial de Doctores y Licenciados en Bellas Artes de Andalucia, 1994, p. 18 y ss., o la tesis de BERMÚDEZ SÁNCHEZ, C. Incidencia del medio en la escultura en madera policromada. Fenómenos físico-químicos. Patologías. Universidad de Granada, 1999

${ }^{8}$ Rosario Marchena Hidalgo, en Las miniaturas de los libros de coro de la catedral de Sevilla: el siglo XVI (Sevilla: Universidad, 1998, p. 32), menciona que se hacen de pino y cedro

${ }^{9}$ DE HAMER, Christopher: Medieval craftsmen: scribes and illuminators, Londres: British Museum Press, 1995, p. 67

${ }^{10}$ Para mas información se puede consultar por ejemplo: TILBROOKE, David. Leather and parchment, composition and structure, IIC Bulletin, col 5, n¹, 1979, p. 9-15

${ }^{11}$ En REBRICOVA, N.L., SOLOVYOVA, N.I. Electron microscopic and biochemical investigation of parchment, ICOM Committee for Conservation: 8th triennial meeten, Sydney 6-11 sep. 1987 (preprints Getty Conservation Institute, 1987, p. 1197-1200. Las moléculas de colágeno consisten en la asociación helicoidal de tres cadenas mediante enlaces por puentes de hidrógeno y enlaces disulfuro y de sus características se sabe que tiene una masa molecular de 300.000 daltons, una longitud de $280 \mathrm{~mm}$ y un grosor de $1.4 \mathrm{~mm}$. JIMENO, Antonio y otros: Biología. Madrid: Ed. Santillana, 1996, p. 84 añade que su peso molecular está próximo a 70.000 $\mathrm{gr} / \mathrm{mol}$

${ }^{12}$ Para obtener más información sobre el proceso de estabilización mediante taninos, consultar las obras de CUNHA, George Martin, TUCKER, N.P. y CUSHING, S. E.: Review of materials, en Library and archives conservation. Massachusetts: The Boston Athenaeum 's 1971 Seminar on the aplication of chemical and phisical methods to the conservation of library and archival materials, Mayo 17-21 1971, p. 28 y el capitulo de Betty M. HAINES en PETHERBRIDGE, Guy: Conservation of Library and Archive Materials and the Graphics Arts, The Institute of Paper Conservation and the Society of Archivists, London: Butterworths, 1987, p. $239-250$
${ }^{13}$ Pueden ser inorgánicos o minerales (alumbre, aluminio, cromo y zirconio), orgánicos (productos naturales fundamentalmente vegetales como la corteza de roble, las vainas de las acacias o las nueces que crean algunos árboles al ser atacados por ciertos insectos) y sintéticos (formaldehido y resinas entre otros)

${ }^{14}$ Proceso que hemos seguido del que Betty M. Haines menciona en PETHERBRIDGE, Guy: Conservation of Library and Archive Materials and the Graphics Arts, The Institute of Paper Conservation and the Society of Archivists, London: Butterworths, 1987, pág. 248-249. Y lo hemos completado con datos que hemos obtenido de Gran Enciclopedia Rialp. Madrid: Ediciones Rialp, 1972, p. $29-31$

${ }^{15}$ Como el sulfuro sódico del 1 al 3\% en agua, añadiendo posteriormente hidróxido cálcico. Son datos que hemos obtenido de Gran Enciclopedia Rialp. Madrid: Ediciones Rialp, 1972, p. $29-31$

${ }^{16}$ MARCHENA HIDALGO, Rosario. Las miniaturas de los libros de coro de la catedral de Sevilla: el siglo XVI. Sevilla: Universidad, 1998. p. 33

${ }^{17}$ Sin embargo esto no se ha conseguido debido a la inadecuada librería en que se encuentran

${ }^{18}$ Se presentan a modo de orientación y porque debían ser similares aunque son de finales del

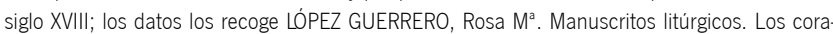
les de la diócesis de Guadix-Baza. La Catedral de Guadix (tesis doctoral) Granada: Facultad de Filosofia y Letras, 1997. La autora aporta los siguientes datos: se pagan 53 reales de vellón por 50 clavos de bronce con el porte, que se han traido de Granada (libro de fábrica mayor-rentas, leg. 25, 20 de marzo de 1786), 42 reales por 12 carzas de clavos de metal con el porte 6 reales desde Granada (libro de fábrica mayor, №11, tomo 1, f.296r 5 de julio de1816), 92 reales por las manezuelas o manillas (hebillas) que hizo para los libros de coro (libro de fábrica mayorrentas, leg. 5, f. 1.24 de diciembre de 1776), 4 reales por 6 juegos completos de cantoneras que hace Álvarez. La mayoría se hacen en Granada pero algunos se traen de Almería como 6 juegos de cantoneras que hace Álvarez y por las que se pagan 274 reales 